\title{
Fruit and vegetable intake and risk of cancer in the Swedish women's lifestyle and health cohort
}

\author{
Marie Löf · Sven Sandin • Pagona Lagiou • \\ Dimitrios Trichopoulos • Hans-Olov Adami • \\ Elisabete Weiderpass
}

Received: 7 April 2010/Accepted: 16 November 2010/Published online: 2 December 2010

(C) The Author(s) 2010. This article is published with open access at Springerlink.com

\begin{abstract}
Objective To investigate whether intake of fruits and vegetables is associated with overall cancer incidence in a large prospective cohort of women in Sweden characterised by young age at enrolment (30-49 years) and relatively low intake of fruits and vegetables.

Methods We followed prospectively 49,261 women, who completed a food frequency questionnaire in 1991-1992. A total of 2,347 incident invasive cancer cases were identified until December 2006. The occurrence of cancer was analysed by fitting Poisson regression models, estimating incidence rate ratios (RR) and 95\% confidence intervals (CI).
\end{abstract}

M. Löf $(\bowtie) \cdot$ S. Sandin · D. Trichopoulos · H.-O. Adami ·

E. Weiderpass

Department of Medical Epidemiology and Biostatistics,

Karolinska Institutet, PO 281, 17177 Stockholm, Sweden

e-mail: marie.lof@ki.se

\section{P. Lagiou}

Department of Hygiene, Epidemiology and Medical Statistics, University of Athens Medical School, 75 M Asias Street, Goudi,

11527 Athens, Greece

P. Lagiou · D. Trichopoulos · H.-O. Adami

Department of Epidemiology, Harvard School of Public Health,

677 Huntington Avenue, Boston, MA 02115, USA

E. Weiderpass

The Cancer Registry of Norway, Montebello,

0310 Oslo, Norway

E. Weiderpass

Department of Community Medicine, University of Tromso,

Tromso, Norway

E. Weiderpass

Folkhälsan Research Center, Samfundet Folkhalsan,

PB 211, 00250 Helsinki, Finland
Results The median intake of fruits and vegetables was $204 \mathrm{~g} /$ day (10th; 90th percentile: 37; $564 \mathrm{~g} /$ day). Intake of fruits and vegetables was not statistically significantly associated with overall cancer incidence. When we compared women in the highest quintile of fruit and vegetable intake to women in the lowest quintile, the RR for overall cancer was 1.01 (95\% CI: 0.88-1.16). Similar results were obtained when investigating the effect of intake of fruits and vegetables separately and when we stratified women by age at follow-up. Conclusion Fruit and vegetable intake was not associated with risk of total cancer in this prospective cohort of women in Sweden.

Keywords Fruits and vegetables - Cancer · Cohort . Epidemiology

\section{Introduction}

Low dietary intake of fruits and vegetables has been suggested to explain $5-12 \%$ of the overall cancer incidence in different populations [1]. A cancer protective effect of fruits and vegetables is biologically plausible, because these foods are rich sources of vitamins, antioxidants, dietary fibre and other compounds presumed to counter tumour development and growth, on account of their antiproliferative and antioxidative potential [2].

The impact of dietary consumption of fruits and vegetables on risk of specific cancers sites has been extensively examined [2]. However, only six reports have evaluated the association between fruit and vegetable intake and overall cancer incidence within prospective cohort studies [3-8], and they have not been able to conclusively establish an inverse association. Firstly, Shibata et al. [6] reported an inverse association between fruit and vegetable intake and 
cancer incidence in US women based on frequency categories of fruits and vegetables in US women, but this study was limited due to poor control of smoking. Thereafter, a pooled analysis of the Health Professionals' Follow-up Study and the Nurses' Health Study [5] as well as an analysis within the Japan Public Health Based Prospective Study reported null associations [7]. In contrast, an analysis within the Greek component of the European Prospective Investigation into Cancer and Nutrition (EPIC) revealed an inverse association between fruit and vegetable intake and cancer incidence [3]. Finally, the two largest studies on the topic have been conducted recently. In the National Institutes of Health-AARP Diet and Health Study (483,338 men and women, 50,863 cancer cases), no support for an association between the intake of fruit and vegetable and the incidence of total cancer was found [4]. However, the EPIC cohort study with data from 10 European countries (478,478 men and women, 30,604 cancer cases) showed a very small inverse association between higher intake of fruits and vegetables in both men and women and overall cancer risk [8].

The studies in this area, with the exception for the analysis within the Nurses' Health Study [5], have been conducted in older populations: age at enrolment ranged between 61-89 years [6], 40-75 years [5], 50-71 years [4], 45-74 years [7] and 25-70 years [9]. There is increasing evidence that lifestyle factors early in life might be particularly important for the risk of breast cancer and probably other malignancies as well. In this regard, the Swedish Women's Lifestyle and Health Cohort Study is unique because the lower age limit at enrolment was 30 years, and the upper was only 50 years. Furthermore, the range of exposure to fruits and vegetables varies considerably across populations, and this might be informative, for example, if only extremely high intake is beneficial or very low intake is harmful. The Swedish population has a segment with relatively low intake of fruits and vegetables $[8,10]$. Furthermore, given the contradictory results of the studies referred to above, the scientific community is facing a major question. Is there a true inverse association attenuated by misclassification? Or is there no association and the findings reflect residual confounding? Our study aims to contribute to the collective evidence that will allow resolution of this important question. Thus, we investigated whether dietary intake of fruits and vegetables is associated with overall cancer incidence in the Swedish Women's Lifestyle and Health Cohort Study.

\section{Subjects and methods}

\section{Study population}

The cohort, described in detail previously, consisted of 49,261 women in the Swedish Women's Lifestyle and
Health Study [11]. Briefly, the study population comprised women aged 30-49 years at recruitment (born 1942-1962) and residing in the Uppsala Health Care Region in 1991-1992. From this population, 96,000 women, randomly selected from four age strata (30-34, 35-39, 40-44 and 45-49 years), were invited to participate. All women were asked to fill in an extensive questionnaire returned by 49,261 who were enrolled in the study. The study has been approved by the ethical committee, Uppsala University, Sweden.

In the present analysis, we excluded 4,423 women due to cancer diagnosed before enrolment $(n=714)$, emigration before the start of the follow-up $(n=7)$, very high or low calculated energy intake $(<1$ st percentile or $\geq 99$ th percentile; $n=1,065$ ) and missing information on body mass index (BMI), education or smoking $(n=2,637)$. Thus, the final analysis was conducted on 44,838 women.

\section{Questionnaire and dietary assessment}

The self-administered questionnaire assessed a large number of lifestyle factors including average intake of foods and beverages [10]. Dietary habits during the 6 months preceding enrolment in the study were ascertained through a validated food-frequency questionnaire covering frequency and quantity of consumption of about eighty food items and beverages [12]. We calculated individual total energy intake by linking the amounts of foods assessed through the questionnaire to the food composition database of the National Food Administration in Sweden [13]. Total intake of vegetables was the sum of broccoli, cabbage, carrot, cauliflower, onion, root, spinach, tomato, pea and bean. Total intake of fruits was the sum of apple, banana, orange and fruit juice. The Spearman correlation coefficient between fruit intake estimated by means of the FFQ and corresponding estimate obtained using dietary records in 111 women was $0.49(p=0.0001)$ [14]. The corresponding Spearman correlation coefficient for vegetables was 0.37 ( $p=0.0001)$ [14].

Lifestyle factors included questions on physical activity, use of supplementary multivitamins, weight and height, alcohol, smoking as well as education, and they referred to the "current situation," i.e., at baseline. For smoking, the women were also asked questions about former smoking habits (defined as 5 years before enrolment in the study).

\section{Follow-up}

Each resident in Sweden is assigned an individually unique national registration number. This number was used to link the data from the cohort with nationwide registers in Sweden, thus allowing virtually complete follow-up of the study women. From the total population registers, we 
received information on the dates of death for women who died during the follow-up period and dates of emigration. Linkage to the national cancer registry, which began in 1958, provided data on prevalent cancer cases at cohort enrolment and on cancers diagnosed in the cohort during follow-up. The outcome studied was any incident invasive cancer except for non-melanoma skin cancer.

The start of follow-up was defined as the date of return of the questionnaire. Observation time was calculated from date of entry into the cohort until the occurrence of incident cancer, emigration, death or end of the observation period (31 December 2006), whichever came first.

\section{Statistical analyses}

The risk of overall cancer was analysed by fitting Poisson models using attained age as time scale [15]. Relative risks were estimated by means of incidence rate ratios (RR) and were considered as statistically significant when the associated two-sided 95\% Wald-type confidence interval did not include the value of one. The goodness of fit of the different models was assessed using the Akaike information criteria (AIC).

For fruits and vegetables combined, we fitted a multivariate model including, categorically, education (0-10, $11-13,>13$ years), body mass index (BMI: $<25,25-29.9$, $\geq 30 \mathrm{~kg} / \mathrm{m}^{2}$ ), smoking [never, former ( $<10$ cigarettes/day), former (10-14 cigarettes/day), former (15-19 cigarettes/ day), former ( $\geq 20$ cigarettes/day), current ( $<10$ cigarettes/ day), current (10-14 cigarettes/day), current (15-19 cigarettes/day) and current ( $\geq 20$ cigarettes/day)], as well as categorically, alcohol intake ( $<5 \mathrm{~g} /$ day, 5-24.9 g/day, $>25 \mathrm{~g} /$ day) and energy intake (in $\mathrm{kJ} /$ day, continuously). Fruit and vegetable intake was then added to the model, alternatively, as: (1) a continuous covariate (in $200 \mathrm{~g} / \mathrm{day}$ increments for fruits and vegetables and in $100 \mathrm{~g} / \mathrm{day}$ increments for fruits as well as for vegetables) (2) in quintiles (3) in deciles (4) through splines. The models utilising splines allow for an informal evaluation of the functional form, for instance, if a linear response function is appropriate. Fruits and vegetables were evaluated as residuals on energy intake [16]. The same models were then repeated for fruits, as well as for vegetables separately.

The covariates in our final models above were chosen based on existing knowledge regarding factors that may confound the association between fruits and vegetables and total cancer risk [2]. Since our outcome was total cancers that include several different cancers with different underlying aetiology, we restricted our analysis to include as few covariates as possible and only the most important ones. Thus, we choose to include education (as a marker of socioeconomic status), body mass index, smoking and alcohol in our final model. The categories for education were based on the Swedish school system in order to represent low, intermediate and high levels of education, while the categories for BMI were based on the WHO classification of under- or normal-weight, overweight and obesity [17]. The alcohol categories corresponded to low, moderate and high intake in this cohort with the purpose to adjust for confounding.

Other potential confounding factors that we considered were multivitamin use (yes or no) and physical activity level $(1,2,3,4$ or 5 , ordered where level 1 corresponds to a sedentary lifestyle, level 3 to a moderately active lifestyle characterised by a few walks per week and level 5 to an active lifestyle with exercise a couple of times per week). In this cohort, $15 \%$ of the women reported to use supplementary multivitamins at baseline. Use of supplementary multivitamins was not associated with overall cancer risk (RR: 0.97, 95\% CI: 0.85-1.10 when comparing users to non-users), and thus this variable was not included in our final model. Having a higher level of physical activity was associated with lower overall cancer risk (RR: 0.76, 95\% CI: 0.59-0.99 when contrasting highest (i.e., 5) to lowest (i.e., 1) level of physical activity). However, since as many as 1,871 women had missing information on physical activity due to power reasons, we did not include physical activity in our final model. Instead, in secondary analysis, we tested whether adjustment for physical activity affected our estimates.

In a series of sensitivity analyses, we also examined whether the risk estimates were changed when we excluded fruit juice from the fruit variable, as well as when excluding very high intake of vegetables and fruits defined as $>500 \mathrm{~g} /$ day and $>1,000 \mathrm{~g} /$ day, respectively. These cutoffs were chosen based upon the composition of a usual Swedish diet [18]. Furthermore, to explore the potential residual confounding by smoking, we conducted sensitivity analyses among never and ever smokers.

Based on the average age at menopause in Sweden being 50 years [19], and the fact that the precise age at menopause was only available for a subset of the cohort, the effects of menopausal status and other risk factors in different periods of life were evaluated by fitting separate models for cancer occurring before and after the age of 50 .

All statistical analyses were carried out using the SAS software version 9.2 (SAS Institute Inc, Cary, NC, USA) and R version 2.5.0 (www.r-project.org).

\section{Results}

The 44,838 women were followed for an average of 14 years $(627,700$ person-years $)$. The baseline characteristics for the women in the entire cohort, as well as per 
quintile of combined fruit and vegetable intake, are shown in Table 1. The prevalence of current smokers was higher in the 1 st and 2 nd than in the 3rd, 4th and 5 th quintiles of fruit and vegetable intake. Furthermore, women in the higher fruit and vegetable quintiles were more educated than women in the lower quintiles. The median intake of fruits and vegetables in this cohort was $204 \mathrm{~g} /$ day with a substantial range of variation from $37 \mathrm{~g} /$ day in the 10th percentile to $564 \mathrm{~g} /$ day in the 90 th percentile. Fruit was the major contributor to the combined category of fruit and vegetable intake. For half of the women, $69 \%$ of the intake of fruits and vegetables was fruit. For a quarter of the women, $78 \%$ of the intake of fruit and vegetable was fruit.

During follow-up, 2,347 incident invasive primary cancer cases were reported to the cancer registry. On average, the cases were 53 years old when diagnosed with cancer. The distribution of the 2,347 cancer cases by site is shown in Table 2. The most frequent primary cancer form was breast cancer ( $46 \%$ of all cancer cases).

Table 3 shows RR for overall cancer with 95\% CIs per quintile of intake of fruits, vegetables, as well as fruits and vegetables combined. There was no statistically significant association between intake of fruits, intake of vegetables or combined intake of fruits and vegetables and overall cancer. Similar results were obtained whether fruit and vegetable intake was fitted as linear continuous variables (Table 3), deciles or as splines (data not shown).

Adjustment for physical activity level did not affect the results in any major way (data not shown). Exclusion of fruit juices from the fruit variable, again, did not materially affect the estimated hazard ratios (data not shown). Finally, excluding very high intake of vegetables ( $>500 \mathrm{~g} /$ day) and fruits $(>1,000 \mathrm{~g} /$ day) from the analyses did not affect any of the observed hazard ratio estimates (data not shown).

Of the 2,347 cancer cases, 985 occurred before the age of 50 years, while 1,362 were diagnosed after the age of 50 years. There was no evidence of effect modification by menopausal status ( $<50$ years, $>50$ years) (data not shown).

When we restricted our analyses to never-smokers, the RRs did not change in any major way. For instance, among never-smokers, when comparing women in the fifth quintile of fruit and vegetable intake with women in the first quintile, the RR was 1.04 (95\% CI: 0.85-1.28).

Similar estimates as those presented in Table 3 were obtained when we investigated only the first 8 years of follow-up (data not shown).

The RR for the combined intake of fruits and vegetables was 0.94 (95\% CI: 0.86-1.03) for a $200 \mathrm{~g} / \mathrm{day}$ increase for breast cancer $(n=1,067)$. The corresponding RR for the

Table 1 Baseline characteristics for the entire cohort at enrolment as well as by quintile of intake of fruits and vegetables for the Swedish women's lifestyle and health study

\begin{tabular}{|c|c|c|c|c|c|c|}
\hline \multirow[t]{2}{*}{ Characteristics } & \multirow[t]{2}{*}{ Entire cohort } & \multicolumn{5}{|c|}{ Total fruit and vegetable quintiles } \\
\hline & & 1 & 2 & 3 & 4 & 5 \\
\hline$N$ & 44,838 & 8,624 & 9,134 & 9,112 & 9,104 & 8,864 \\
\hline Age, mean \pm SD (years) & $39 \pm 6$ & $38 \pm 6$ & $39 \pm 6$ & $39 \pm 6$ & $40 \pm 6$ & $40 \pm 6$ \\
\hline \multicolumn{7}{|l|}{ BMI, $n(\%)$} \\
\hline$<25 \mathrm{~kg} / \mathrm{m}^{2}$ & $32,529(73 \%)$ & $6,300(73 \%)$ & $6,704(73 \%)$ & $6,687(73 \%)$ & $6,609(73 \%)$ & $6,229(70 \%)$ \\
\hline $25-30 \mathrm{~kg} / \mathrm{m}^{2}$ & $9,731(22 \%)$ & $1,748(20 \%)$ & $1,950(21 \%)$ & $1,926(21 \%)$ & $2,016(22 \%)$ & $2,091(24 \%)$ \\
\hline$\geq 30 \mathrm{~kg} / \mathrm{m}^{2}$ & $2,578(6 \%)$ & $576(7 \%)$ & $480(5 \%)$ & $499(6 \%)$ & $479(5 \%)$ & $544(6 \%)$ \\
\hline \multicolumn{7}{|l|}{ Education, $n(\%)$} \\
\hline Up to 10 years & $13,250(30 \%)$ & $3,342(39 \%)$ & $2,903(32 \%)$ & $2,485(27 \%)$ & $2,345(26 \%)$ & $2,175(24 \%)$ \\
\hline $11-13$ years & $17,589(39 \%)$ & $3,413(40 \%)$ & $3,640(40 \%)$ & $3,654(40 \%)$ & $3,528(39 \%)$ & $3,354(38 \%)$ \\
\hline$>13$ years & $13,999(31 \%)$ & $1,869(22 \%)$ & $2,591(28 \%)$ & $2,973(33 \%)$ & $3,231(35 \%)$ & $3,335(38 \%)$ \\
\hline \multicolumn{7}{|l|}{ Smoking, $n(\%)$} \\
\hline Current & $13,059(29 \%)$ & $3,404(40 \%)$ & $2,853(31 \%)$ & $2,502(27 \%)$ & $2,275(25 \%)$ & $2,025(23 \%)$ \\
\hline Former & $13,274(30 \%)$ & $2,295(27 \%)$ & $2,638(29 \%)$ & $2,606(29 \%)$ & $2,831(31 \%)$ & $2,904(33 \%)$ \\
\hline Never & $18,505(41 \%)$ & $2,925(34 \%)$ & $3,643(40 \%)$ & $4,004(44 \%)$ & $3,998(44 \%)$ & $3,935(44 \%)$ \\
\hline \multicolumn{7}{|l|}{ Alcohol intake, $n(\%)$} \\
\hline$<5$ g/day & $33,548(74.8 \%)$ & $6,654(77 \%)$ & $6,774(74.6 \%)$ & $6,778(74.3 \%)$ & $6,718(73.7 \%)$ & $6,624(74.7 \%)$ \\
\hline $5-25$ g/day & $11,096(24.8 \%)$ & $1,919(22 \%)$ & $2,316(24.9 \%)$ & $2,301(25.3 \%)$ & $2,354(25.9 \%)$ & $2,206(24.9 \%)$ \\
\hline$>25 \mathrm{~g} /$ day & $194(0.4 \%)$ & $51(1 \%)$ & $44(0.5 \%)$ & $33(0.4 \%)$ & $32(0.4 \%)$ & $34(0.4 \%)$ \\
\hline Energy, mean $\pm \mathrm{SD}(\mathrm{kJ} /$ day $)$ & $6,520 \pm 1,870$ & $6,840 \pm 2,070$ & $6,390 \pm 1,830$ & $6,340 \pm 1,780$ & $6,400 \pm 1,760$ & $6,620 \pm 1,840$ \\
\hline
\end{tabular}

$S D$ standard deviation 
Table 2 Distribution of the 2,347 incident cancer cases among the 44,838 women in the Swedish women's lifestyle and health study

\begin{tabular}{|c|c|c|c|}
\hline ICD-7 & Cancer site & Cases $(\mathrm{N})$ & $\%$ \\
\hline 170 & Breast & 1,067 & 45.5 \\
\hline 190 & Malignant melanoma of the skin & 153 & 6.5 \\
\hline 175 & Ovary & 122 & 5.2 \\
\hline 162 & Lung (including bronchus and trachea) & 118 & 5.0 \\
\hline 193 & Brain and other central nervous system & 114 & 4.9 \\
\hline 172 & Corpus uteri & 112 & 4.8 \\
\hline 153 & Colon & 80 & 3.4 \\
\hline 195 & Endocrine glands other than thyroid & 65 & 2.8 \\
\hline 171 & Cervix uteri & 63 & 2.7 \\
\hline 154 & Rectum & 48 & 2.1 \\
\hline 200 & Lymphosarcoma & 39 & 1.7 \\
\hline 180 & Kidney & 38 & 1.6 \\
\hline 181 & Bladder and other urinary tract & 30 & 1.3 \\
\hline 194 & Thyroid gland & 26 & 1.1 \\
\hline 151 & Stomach & 24 & 1.0 \\
\hline 157 & Pancreas & 22 & 0.9 \\
\hline 197 & Connective tissue & 19 & 0.8 \\
\hline 176 & Other unspecified female genital organs & 16 & 0.7 \\
\hline 203 & Multiple myeloma & 16 & 0.7 \\
\hline 204 & Leukaemia and aleukemia & 16 & 0.7 \\
\hline 205 & Mycosis fungoides & 15 & 0.6 \\
\hline 174 & Uterus unspecified & 14 & 0.6 \\
\hline \multirow[t]{2}{*}{155} & Liver & 14 & 0.6 \\
\hline & Other cancers & 116 & 5 \\
\hline
\end{tabular}

combined intake of fruits and vegetables and all other cancers excluding breast cancer $(n=1,280)$ was 1.04 (95\% CI: 0.96-1.12). Correspondingly, there was no statistically significant association between intake of fruits or vegetables for breast cancer or all other cancers (excluding breast cancer).

\section{Discussion}

Fruit and vegetable intake was not associated with risk of total cancer in this prospective cohort of women in Sweden. This finding is not consistent with the recent multicentre analysis within the EPIC cohort study [8] that reported a weak, but statistically significant, inverse association between fruit and vegetable intake and overall cancer risk [8]. However, their analysis included nearly 30,000 cases $(21,000$ female ones), and it is the second largest study that has evaluated fruit and vegetable intake in relation to total cancer incidence [8]. Thus, they had a substantially higher power than this cohort. If the true magnitude of the overall cancer protective effect of fruits and vegetables is modest, our study may not have had the statistical power to detect this; however, our results are in line with analyses within four other cohorts [4, 5, 7].

The Greek component of the EPIC cohort study reported an inverse association between higher intake of fruits and vegetables and cancer incidence in women. That analysis relied on only 430 cases of cancer among women [3]. In the

Table 3 Relative risk of overall cancer estimated as incidence rate risk ratios (RR) with two-sided 95\% confidence intervals (CIs) for fruit and vegetable intake, fruit intake only and vegetable intake only

\begin{tabular}{|c|c|c|c|c|c|c|c|}
\hline & \multicolumn{5}{|c|}{ Quintiles } & \multirow[t]{2}{*}{ Continuous intake ${ }^{a}$} & \multirow[t]{2}{*}{ P-value } \\
\hline & Q1 & Q2 & Q3 & Q4 & Q5 & & \\
\hline \multicolumn{8}{|c|}{ Vegetables and fruits } \\
\hline Median, g/day & 88 & 146 & 198 & 263 & 395 & & \\
\hline Cases & 445 & 454 & 479 & 450 & 519 & & \\
\hline $\mathrm{RR}(95 \% \mathrm{CI})^{\mathrm{b}}$ & 1.00 & $0.98(0.85-1.13)$ & $0.99(0.86-1.14)$ & $0.89(0.77-1.03)$ & $1.01(0.88-1.16)$ & $0.99(0.93-1.05)$ & 0.81 \\
\hline \multicolumn{8}{|l|}{ Fruits } \\
\hline Median, g/day & 47 & 94 & 134 & 184 & 288 & & \\
\hline Cases & 473 & 449 & 462 & 471 & 492 & & \\
\hline $\mathrm{RR}(95 \% \mathrm{CI})^{\mathrm{b}}$ & 1.00 & $0.97(0.84-1.11)$ & $0.94(0.81-1.08)$ & $0.92(0.80-1.06)$ & $0.97(0.84-1.12)$ & $0.99(0.95-1.03)$ & 0.50 \\
\hline \multicolumn{8}{|l|}{ Vegetables } \\
\hline Median, g/day & 36 & 51 & 62 & 75 & 98 & & \\
\hline Cases & 418 & 472 & 477 & 448 & 532 & & \\
\hline $\operatorname{RR}(95 \% \mathrm{CI})^{\mathrm{b}}$ & 1.00 & $1.04(0.90-1.20)$ & $1.05(0.91-1.22)$ & $0.97(0.84-1.12)$ & $1.09(0.85-1.25)$ & $1.02(0.96-1.08)$ & 0.57 \\
\hline
\end{tabular}

${ }^{a}$ Per $200 \mathrm{~g} /$ day for fruits and vegetables, per $100 \mathrm{~g} /$ day for fruits and per $100 \mathrm{~g} /$ day for vegetables

b Adjusted for education, BMI, smoking, energy and alcohol intake. Attained age was utilised as time scale 
Greek cohort, the median intake of fruits and vegetables (including legumes) was $837 \mathrm{~g} /$ day [3]. This figure is much higher than in our cohort (median intake $204 \mathrm{~g} /$ day), as well as in earlier prospective cohorts $[4,5,8]$. Hence, there may be a biological threshold for the cancer protective effect of fruits and vegetables. Furthermore, anticancer effects may also differ between types of fruits and vegetables, and the distribution of intake likely varies between the populations. The Greek diet is characterised by higher intake of legumes than US and Northern European diets, and it also likely consists of more fresh seasonal vegetables and fruits. In this study, we did not find any evidence for that the low fruit and vegetable intake in this population were harmful in terms of increased overall cancer risk. In this context, it is relevant to note that the multicentre EPIC analysis could not detect statistically significant heterogeneity among countries, although absolute intake between the investigated countries varied greatly (with higher intake in southern European countries such as Greece and lower intake in northern European countries such as Sweden) [8].

In our study, we assessed fruit and vegetable consumption only once, entailing misclassification among those who changed their dietary pattern during follow-up. Because it is likely to be non-differential, such misclassification attenuates the strength of any true association. However, there is a potential risk that the degree of this misclassification increases over time, because women change their dietary habits. Thus, the longer the follow-up, the more attenuated the association might be. However, for 0-8 years follow-up, the results were similar as for the complete follow-up ( $0-14$ years), and thus no evidence for such an attenuation was observed.

Both EPIC and the National Institutes of Health-AARP cohorts assessed residual confounding due to smoking $[4,8]$. In this study, the results of the restricted analysis to never-smokers argue against substantial residual confounding by smoking.

Our study has some limitations. Consumption of fruits and vegetables might be underestimated in our study, because intake of only 10 vegetables and four fruits was assessed in our FFQ. However, the estimated fruit and vegetable intake in our cohort is similar to that in the Swedish component of the EPIC cohort [8], another Swedish cohort [20] as well as in a nationwide survey from 1997 [18]. The latter used 7-day food diaries in order to assess dietary intake. These diaries are considered to be superior to FFQs in assessing actual current levels of intake, and they often produce slightly higher estimates. There is also a risk that fruit and vegetable intake may be overestimated since these foods are considered healthy, but considering the low intake overall and that the intake agreed with other dietary data in Swedish women $[8,18,20]$, it is less likely. Misclassification of fruit and vegetable intake due to measurement error (whether underestimation or overestimation) associated with the food frequency questionnaire is unavoidable, but, given the prospective study design, it is likely to be non-differential and thus could attenuate any true association. Another limitation is that our study was too small to separately examine the relationship between fruit and vegetable intake and cancer at specific sites. Strengths of our study include its prospective design, relatively large size and complete follow-up. Cancer registration in Sweden is mandatory, making ascertainment of incident cases virtually complete. Furthermore, we were able to adjust for possible confounding variables such as smoking, alcohol and multivitamin supplement use.

Almost half of all cancers in our cohort were breast cancer, while the prevalence of colorectal cancer was low $(5 \%)$. Our results are reasonable considering the conclusions in the recent World Cancer Research Fund (WCRF) report [2] that the evidence for a protective effect of fruits and vegetables is probable for colorectal cancer and limited for breast cancer. Hence, with the prevailing distribution of cancer sites and types among young and middle-aged women in Sweden and many other western countries, the results from this and most other prospective cohorts do not suggest that increased fruit and vegetable intake reduces overall cancer risk in this segment of the population. Still, our results are relevant in the context of understanding cancer aetiology. Moreover, they do not contradict current public health recommendations, because high intake of fruits and vegetables may protect from specific types of cancer, mostly in the gastrointestinal tract [2], as well as from cardiovascular disease [5]. Furthermore, fruits and vegetables are important sources of dietary fibre, essential vitamins and minerals and facilitate, due to their low energy content, the maintenance of a healthy body weight which is conducive to a lower overall cancer incidence [21-23].

In conclusion, fruit and vegetable intake was not associated with risk of total cancer in this prospective cohort of Swedish women.

Acknowledgments This study was funded by the Swedish Research Council, Swedish Cancer Society, Swedish Council for Planning and Co-ordination of Research and National Cancer Institute.

Conflict of interest There is no conflict of interest.

Open Access This article is distributed under the terms of the Creative Commons Attribution Noncommercial License which permits any noncommercial use, distribution, and reproduction in any medium, provided the original author(s) and source are credited.

\section{References}

1. Vainio H, Weiderpass E (2006) Fruits and vegetables in cancer prevention. Nutr Cancer 54:111-142 
2. World Cancer Research Fund (2007) Food, nutrition and physical activity and the prevention of cancer. A global perspective. American Institute for Cancer Research, Washington, DC

3. Benetou V, Orfanos P, Lagiou P, Trichopoulos D, Boffetta P, Trichopoulou A (2008) Vegetables and fruits in relation to cancer risk: evidence from the Greek EPIC cohort study. Cancer Epidemiol Biomarkers Prev 17:387-392

4. George SM, Park Y, Leitzmann MF et al (2009) Fruit and vegetable intake and risk of cancer: a prospective cohort study. Am J Clin Nutr 89(1):347-353

5. Hung HC, Joshipura KJ, Jiang R et al (2004) Fruit and vegetable intake and risk of major chronic disease. J Natl Cancer Inst 96(21):1577-1584

6. Shibata A, Paganini-Hill A, Ross RK, Henderson BE (1992) Intake of vegetables, fruits, beta-carotene, vitamin $C$ and vitamin supplements and cancer incidence among the elderly: a prospective study. Br J Cancer 66(4):673-679

7. Takachi R, Inoue M, Ishihara J et al (2008) Fruit and vegetable intake and risk of total cancer and cardiovascular disease: Japan public health center-based prospective study. Am J Epidemiol 167(1):59-70

8. Boffetta P, Couto E, Wichmann J et al. (2010) Fruit and vegetable intake and overall cancer risk in the European prospective investigation into cancer and nutrition (EPIC). J Natl Cancer Inst 102(8):1-9. Advance Access published on April 6, 2010. doi: 10.1093/jnci/djq072

9. Riboli E, Hunt KJ, Slimani N et al. (2002) European prospective investigation into cancer and nutrition (EPIC): study populations and data collection. Public Health Nutr 5(6B):1113-1124

10. Lagiou P, Trichopoulos D, Sandin S et al (2006) Mediterranean dietary pattern and mortality among young women: a cohort study in Sweden. Br J Nutr 96(2):384-392

11. Kumle M, Weiderpass E, Braaten T, Persson I, Adami HO, Lund E (2002) Use of oral contraceptives and breast cancer risk: the Norwegian-Swedish women's lifestyle and health cohort study. Cancer Epidemiol Biomarkers Prev 11(11):1375-1381
12. Wolk A, Bergstrom R, Hunter DJ et al (1998) A prospective study of association of monounsaturated fat and other types of fat with risk of breast cancer. Arch Intern Med 158:41-45

13. National Food Administration (1989) Food composition tables. National Food Administration, Uppsala, Sweden

14. Khani B, Ye W, Terry P, Wolk A (2004) Reproducibility and validity of major dietary patterns among Swedish women assessed with a food-frequency questionnaire. J Nutr 134:1541-1545

15. Korn EL, Graubard BI, Midthune D (1997) Time-to-event analysis of longitudinal follow-up of a survey: choice of the timescale. Am J Epidemiol 145(1):72-80

16. Willett W, Howe GR, Kushi LH (1997) Adjustment for total energy intake in epidemiologic studies. Am J Clin Nutr 65: 1220S-1228S

17. World Health Organisation (WHO) (2000) Obesity: preventing and managing the global epidemic. Report of a WHO consultation. Technical Report Series no 894. WHO, Geneva

18. Becker W, Pearson M (2002) Riksmaten 1997-1998. Kostvanor och näringsintag i Sverige.Metod och resultatanalys. National Food Administration, Uppsala, Sweden

19. Weiderpass E, Baron J, Adami HO (1999) Low-potency oestrogen and risk of endometrial cancer: a case-control study. Lancet 353:1824-1828

20. Rashidkhani B, Lindblad P, Wolk A (2005) Fruits, vegetables and risk of renal cell carcinoma: a prospective study of Swedish women. Int J Cancer 113(3):451-455

21. Bergstrom A, Pisani P, Tenet V, Wolk A, Adami HO (2001) Overweight as an avoidable cause of cancer in Europe. Int $\mathrm{J}$ Cancer 91(3):421-430

22. Calle EE, Rodriguez C, Walker-Thurmond K, Thun MJ (2003) Overweight, obesity, and mortality from cancer in a prospectively studied cohort of U.S. adults. N Engl J Med 348(17):1625-1638

23. Renehan AG, Soerjomataram I, Tyson M et al (2010) Incident cancer burden attributable to excess body mass index in 30 European countries. Int J Cancer 126(3):692-702 